\title{
Challenges for home care nurses in providing quality care*
}

Karen A. Luker School of Nursing, Midwifery \& Social Work, University of Manchester, Manchester, UK

\begin{abstract}
The purpose of this article is to explore the processes that enable home care nursing services to provide good quality care. The focus is on how quality care is defined by nurses, patients and carers, and palliative care provision is used as an exemplar. The international policy scene is one of escalating costs and shortage of qualified nurses, which has resulted in a variety of forms of skill mix. In addition there has been an emphasis on partnership working with patients and carers, who are encouraged to participate in care giving. The article draws upon data generated in studies conducted by the author and colleagues over a 10-year period. The main conclusions of the article are that nurses, patients and carers were able to articulate the essential components of high quality care and that these elements fitted the structure, process and outcome framework first described by Donabedian (1969). In addition, forming a good relationship and rapport with the patient and family is viewed as a critical pre-requisite to quality care. It is noteworthy that the awareness contexts first described by Glaser and Strauss (1965) are still a relevant framework for analysing nurse patient and carer interactions. Finally it is suggested that the quality indicators identified in this article could be used as a useful starting point to develop criteria for judging quality of provision in palliative and supportive care services.
\end{abstract}

Key words: community nursing; home care nursing; palliative care; quality of care; research programme

Received: January 2006; accepted: March 2006

\section{Introduction}

The aim of this article is to explore the processes that enable home care nursing services to provide good quality care. The article, in the context of developed countries, will illustrate how quality care is defined by nurses, patients and carers, and will use palliative and supportive care provision as the exemplars. Due to the centrality of 'quality' in the provision of care, and the ethical implications of seeking user's views, this article, whilst focussing

\footnotetext{
*This article was first presented as a plenary address at the $3 \mathrm{rd}$ International Conference on Community Health Nursing Research held in Tokyo, Japan in September 2005.
}

Address for correspondence: Karen A. Luker, Queen's Nursing Institute Professor of Community Nursing, Head of School, School of Nursing, Midwifery \& Social Work, University of Manchester, Coupland III Building, Coupland Street, Manchester M13 9PL. Email: Karen.A.Luker@manchester.ac.uk (C) 2006 Cambridge University Press on provision of care to sick people in their own homes, will also have relevance and transferability to other community or home care settings.

\section{Background}

The past two decades have seen many changes in the way that home care nursing services have been organized and configured. These changes in developed countries have been driven largely by the proportional increase in the population of older people requiring long-term health care. Nowhere is this more apparent than in Japan where it is predicted that a quarter of the population will be aged 65 years or older by 2010 . In addition, advances in medical science, mean that people of all ages can be discharged from hospital quicker and sicker and have their follow-up care provided at home by nurses. In many countries there is a shortage of 
nurses in the labour market, which has taxed the imagination of many planners and service providers. In some countries community nursing is not seen as a particularly attractive career option, and there has been an emphasis on the development of other service providers to fill the care gap (eg voluntary organizations). The result of these challenges has been an escalation of costs, and an attempt to contain costs and retain quality of care by redesigning service provision through eligibility criteria and a variety of forms of skill mix. Skill mix is used variously in the literature, but normally implies either enhancement of a job, by extending roles and skills, or some form of substitution and working across professional boundaries. However, there have been few studies that have documented the effectiveness and efficiency of most types of skill mix change and whilst skill mix is perceived as inevitable in terms of providing the service, the jury is still out with respect to its value.

Within the community nursing service skill mix change can be introduced via the following means (drawn from Sibbald et al., 2004):

- Enhancement: that is increasing the depth of a job by extending the role or skills of a particular grade of nurse or care worker. For example, enabling home helps to provide personal care for elderly people.

- Substitution: this implies expanding the breadth and depth of a job, in particular by working across professional divides or exchanging one type of worker for another. For example, nurse practitioners in the USA substitute for doctors in many situations.

- Delegation: this involves moving a task up and down a traditional unidisciplinary ladder. For example, allowing nursing assistants to do wound dressings.

- Innovation: this implies creating new jobs or roles by introducing a new type of worker. For example, home chemotherapy nurse specialist.

- Transfer: this involves moving the provision of a service from one health care sector to another. For example, substituting community for hospital.

- Location: this involves moving the venue from which a service is provided from one health sector to another without changing the people who deliver the service. For example, hospice staff providing palliative care in the home (hospice at home).
- Liaison: namely using specialists in one health care sector to educate and support staff working in another sector. For example, continence advisors who work in hospital clinics providing advice to home care nurses.

\section{Partnership working}

In addition, there has been an emphasis in community nursing on partnership working with patients and carers, who are encouraged to participate in care giving; this is most apparent in the care of older people and palliative care. There is a clear policy emphasis on providing more care for the sick and dying and frail older people in their own homes and this is evident in most developed countries, home is the preferred care location for many patients and is thought to be a cheaper alternative than hospital care. But what is the real cost to carers? In the context of many countries there is a reported problem of caregiver burden and stress and several attempts have been made to develop strategies to reduce the burden of informal care giving. Japan launched a long-term care insurance system in a courageous attempt to reduce the burden of care for frail elderly people. Services are allocated on the basis of the Government-Certified Disability Index, however it is likely that the needs of demented elderly people are underestimated by the system (Ohrui, 2004). In the UK the introduction of skill mix in community nursing means traditional nursing work has been redefined and is now provided by other workers from the social rather than health care sector; this is a growing trend throughout Europe. This means that it is no longer within the personal remit of a qualified community nurse to 'waste valuable resources' on tasks such as washing and bathing patients. Whilst in theory these tasks are delegated to other agencies, tests of eligibility for the services and the need for payment may mean that the burden falls upon the individual carer rather than the state. Lay carers of terminally ill people have been reported to be dissatisfied with the services they have received in the community. Our work (Beaver et al., 1999) indicates that the lay carers of terminally ill people do a substantial amount that is the majority of the nursing work related to maintaining the patient at home (Table 1). 
Table 1 Bereaved carers commenting on patients who were completely unable to carry out activities of daily living $(n=19)$

\begin{tabular}{llll}
\hline ADL & $\begin{array}{l}\text { Patients unable to carry out } \\
\text { activity number }\end{array}$ & $\begin{array}{l}\text { Lay carer carried out } \\
\text { activity number }\end{array}$ & $\begin{array}{l}\text { District nurse carried out } \\
\text { activity number }\end{array}$ \\
\hline Making meals & 16 & 16 & 0 \\
Bath/shower & 15 & 11 & 4 \\
Getting dressed & 12 & 10 & 2 \\
Washing self & 7 & 7 & 0 \\
Mobility & 7 & 7 & 0 \\
Getting to toilet & 7 & 6 & 2 \\
Personal care & 7 & 5 & 0 \\
Getting in/out of bed & 6 & 6 & 0 \\
Eating and drinking & 0 & 0 & \\
\hline
\end{tabular}

Reproduced from Beaver et al. (1999) with kind permission of Mark Allen Group.

\section{Quality and context}

In an effort to improve the quality of health care, professional practice has come under scrutiny and serious questions are now being asked concerning care input and patient outcomes. Practitioners are under an implicit and sometimes explicit obligation to demonstrate that they are using the most up-to-date knowledge and there is now an expectation that practice is informed by research as well as practice-based knowledge. In home care nursing this has brought about a major paradigm shift. In the past home care nurses have relied on personal knowing of the patient, this is no longer possible due to structural changes in the way the service is configured (skill mix) and the fact that home care is centre stage rather than marginal. The role of qualified community nurses is often that of case manager rather than care provider and this has involved a major change in work type. At the heart of nursing work is the quality of the relationship between the nurse and patient (Luker et al., 2000). There is a considerable body of knowledge which attempts to describe this relationship and its therapeutic potential in the caring process. One element of this process namely 'knowing the patient' has received considerable attention in the literature and there are many interpretations of how nurses come to know patients. Based on in-depth interviews and participant observation with a range of nurses working in the community, Speed and Luker (2004) report that the way in which British home care nurses know their patients has changed: direct involvement by the nurse has been replaced by a knowing about or a knowing by proxy. This, it is suggested, has made home care work significantly more difficult, in that second hand knowing effectively distances the nurse from the patients, and gives primacy to techno-care and scientific knowledge hence relegating personal knowing to a secondary position. The impact that this change has on work satisfaction is largely unknown.

\section{Providing quality care}

Providing high quality care has dominated nursing and policy debates for decades. Despite the activity in the field of quality improvement there is little reliable evidence regarding home care nurses' perceptions and definition of quality provision. Indeed with a few notably exceptions the work of home care nurses is largely invisible and is generally poorly described. In addition, the push towards collaborative working and the proliferation of specialist roles, for example in the cancer and palliative care field, means that it remains imperative for home care nurses to demonstrate their value.

We undertook a study to elicit factors associated with the provision of high quality or poor nursing care using a critical incident technique, this paper will focus on the findings from the palliative care data related to this work (Austin et al. (2000), reproduced with kind permission of BMJ Publishing Group). Palliative care services within the UK are provided by both the voluntary and statutory sector and are heavily reliant on carer input. 


\section{Palliative care}

Over the last decade there has been an increasing emphasis on collaborative working between sectors to ensure integration of services. Care provision varies according to geographical location, although the current impetus is for equal access to services for patients regardless of where they live. Services commonly employed in the provision of palliative care include: hospital palliative care teams, primary care teams including home care nurses, hospices, which offer short stays for symptom control, specialist nurses (Macmillan nurses) who offer advice on symptom control to other healthcare professionals and support to patients and carers, and Marie Curie nurses who provide nursing care for patients at home especially at night so that carers are able to rest. We included 67 community/home care nurses of various grades in our study. Suffice it to say here that quality was conceptualized in a multidimensional way and nurses described the situations in which high quality care could be provided, the actions required to provide good quality care, and the indicators that suggested that the desired level of care had been achieved. These characterizations are analogous to Donabedian's (1969) structure, process and outcome categories which have been widely deployed in health care evaluations. We suggest that the structure, process and outcome factors identified in our study could be used as benchmarks for quality review of palliative care services provided by home care nurses and others, we hope that these findings will have some transferability for an international audience.

\section{Structural factors influencing quality}

\section{Early referral of patients}

The early referral of patients to the community/home nursing service whilst beyond the control of the nurse was viewed as an essential pre-requisite if the best care was to be provided. Early referral facilitates contact with the family, who may be viewed as co-workers, and ensures that the community/home nurse can make an assessment of the patient's and family's needs and make suggestions as to the kind of support they might wish to receive. A typical comment from a home/community nursing sister was:
I like to get to know them while they are reasonably well really and build up a relationship then with them. And when they do become terminally ill they tend to accept more and trust you and see that they do know you and you do know them.

Situations in which the community nursing service had not had early contact with the patient could present difficulties and intervening in a crisis situation was viewed negatively by nurses.

\section{Family circumstances}

Family circumstances played a central role, nurses referred to homes as 'good to work in'. A good home was one where the family was actively involved in the patient's care and receptive to the home/community nurse and other services and, importantly, were willing to adapt as the patient's circumstances varied. Relationships within the family also played a notable role. Patients who lived with or had support from family members were deemed more likely to receive better care. This was in part a practical consideration, with somebody being there to help the patient with physical needs and to provide services such as nutritious meals. Family presence was also thought to contribute to patients' general well being by providing emotional support, which enabled patients to stay at home in familiar surroundings if this was their wish.

Conversely, difficulties were encountered if there appeared to be some kind of friction within the family or if the family was hostile to the home/community nursing and other services. Care provision was particularly problematic in situations in which the patient or family appeared to be unaware of the diagnosis or prognosis, not least because of the implications with regard to drawing in other support services. The importance of who knows what in a palliative care situation cannot be underestimated. Getting to know the patient and family in the context of the home involves finding out who knows what. Glaser and Strauss (1965) in their seminal work on the awareness of dying in a hospital context in the USA identified four awareness contexts that fit the community/home setting (Box 1).

The relevance of the awareness contexts to nursing patients is not a new observation. In our 


\section{Box 1 The four awareness contexts}

Closed awareness: 'The situation where the patient does not recognize his impending death even though everyone else does.'

Suspected/suspicion awareness: 'The situation where the patient suspects what others know and therefore attempts to confirm or invalidate his suspicion.'

Mutual pretence awareness: 'The situation where each party defines the patient as dying, but each pretends that the other has not done so.'

Open Awareness: 'The situation where personnel and patient both are aware that he is dying, and where they act on this awareness relatively openly.'

Reproduced from Journal of Advanced Nursing 31, 779 with kind permission of Blackwell Publishing Ltd.

study it was universally the case that nurses found it less stressful and more rewarding to work in a situation of 'open awareness' where both patient and relative/carer were aware of the impending death. These circumstances facilitated the provision of the best quality of care, since time could be invested in assisting patients and carers to cope with the impending loss, rather than undertaking work to prepare the patient and family for receipt of bad news as the following extract highlights:

He told me a little bit about the background of his wife and how long it had been going on and, you know, he was very open and that were easier. It's easier when the situation is open ... but if somebody comes and meets you at the door by saying 'we know what she's got but she doesn't know what she has got and don't tell her'. This obviously it's more difficult.

Caring was also more difficult if relatives were willing but unable to provide care, or felt unable to cope. For example, in situations where relatives lived some distance from the patient or had other commitments. In crisis situations patients could be admitted to hospital or hospice care, and where this decision conflicted with the patient's wishes nurses would consider that they had been unable to provide quality care.

\section{Availability of time}

A considerable influence on the quality of care as perceived by the nurses was the amount of time available. However home/community nurses were at pains to point out that even if they were short of time they tried not to appear rushed in the patient's home. The consequences of this were that other patients missed out, or additional demands were placed on colleagues. There was a shared view amongst nurses that the needs of patients requiring palliative care came first and that, at the very least, the patient's physical needs should be met. In fact, nurses often referred to care that they provided which might be considered to be 'over and above' that required for the patient's immediate nursing needs; for example, tailoring the working day so that the patient could be visited at a specific time. Forming a relationship with the family, making time available and carrying out all aspects of patient care were highly prized by nurses, and were sources of work satisfaction. This was linked in some way to the erosion of their hands-on patient care role with other categories of patient. In proximity to death nurses felt able to justify their personal intervention and interactions with patients, the provision of which was viewed as holistic care. A typical response was:

I haven't got a problem when it comes to nursing terminally ill patients. I find that quite rewarding really because it's the one time as a community nurse you're back doing the basics hands on. That's the only time we do basic hands on general care. So yeah, I do, it's awful to say but I enjoy getting a terminally ill patient because everyone becomes involved. You get to know the patient.

The above extract from the data points up the importance, once again, of getting close to and knowing the patient.

\section{Availability of equipment and services \\ Equipment}

The dependence on equipment had particular relevance; given the general aim of the home/community nursing service is to provide care to patients in their own homes. Whilst much of the equipment required was usually available, difficulties could arise. The provision of equipment was particularly 
problematic if it was required at short notice, at the weekend or in a crisis situation, as access to equipment out-of-hours was less straightforward.

\section{Services}

In order to be allowed to die in their own home sometimes individuals need an enhanced service, over and above that which could be routinely provided by the home/community nursing service. If somebody in the last days of their life required a night sitter (a service provided by the Marie Curie Charity) and one is not available because of high demand then nurses feel that they are unable to provide a quality service. Whilst many of the nurses we interviewed considered that they had expertise in palliative care, specialist nurses (Macmillan nurses) were used as a resource of specialist advice or support. In the main this was welcomed, although some nurses considered that their use could lead to duplication of effort or lack of continuity of care because of the number of personnel involved.

The role of the family doctor (general practitioner, GP) was considered critical in determining how successfully the patient could be cared for at home. Many nurses mentioned their positive working relationships with GPs although, as already noted, difficulties could be encountered if patients were not referred at an early stage. In general GPs did not appear to be routinely involved in the day-today care of patients and were likely to be contacted by the nurse when the patient required some kind of symptom control most commonly a prescription for analgesia. Problems only arose if GPs viewed the need for symptom control differently from either the nurse or patient. A number of nurses recalled incidents where this discrepancy had been distressing for the patient and had led the nurse to feel that the quality of care was not optimum.

\section{Process factors influencing quality}

Care processes largely hinged on the ability to form a positive relationship with the patient, family, other heath care professionals and formal carers.

\section{Relationship with patient and family}

It was considered to be good practice to ensure continuity of care for the patient by keeping the number of community nurses involved to a minimum. This enabled staff to establish a good rapport with the family and aided the establishment of trust between the nurse and family. This relationship aided the planning and provision of care and helped to ensure that the care provided was consistent with the wishes of the patient and family. Indeed, a great deal of emphasis was placed by all grades of staff on caring for the family as well as the patient. Anything which could be done to alleviate the distress of carers was valued by nurses. In some cases this necessitated nurses 'managing the environment' by contriving situations in which they could speak to family members when the patient was not present. Care of the family often extended beyond the time of death in the form of attendance at the patient's funeral and bereavement visits.

\section{Relationships with other health care professional}

The relationship in place with other healthcare providers and formal carers influenced the provision of care. If a good relationship between the community nursing service and other agencies could be maintained, this made it easier to introduce support from these services when appropriate. In this respect the community nursing service seemed to act as the 'lynch pin' or 'gatekeeper' to services provided by other professionals. This was explicitly mentioned by a number of nurses in our study, who viewed themselves as care co-coordinator or manager. The importance of good communication leading to a successful multidisciplinary approach was stressed:

It was nice how all the services pulled together. And within a day we had all the equipment that we needed. The rise and fall bed, hoist, everything in the house. We had the hospice at home team in offering sits. We had Marie Curie involved. We also had rapid response involved. This all took over a period of a fortnight before he died, but everyone worked really very closely together and it worked very well ... good coordination, good communication. Everybody let each other know what they could do, what input they could provide, and it was all coordinated from the community nursing service. 


\section{Outcomes indicating quality}

Our study demonstrated that nurses viewed the primary outcome of palliative care to be maintaining the patient's quality of life up to the time of death. Patients who retained control over their circumstances were cited as positive examples, and death was seen as more acceptable if patients were painfree, comfortable and had their dignity retained. Ultimately the desired outcome was a patient who died a peaceful death, in the place of their choice, supported by family and or friends. Alongside this families who felt supported and able to cope when caring for a dying relative were also given as examples of cases where good care had been achieved. Other indicators related to the feelings of staff. It was not uncommon for nurses to cite examples of where they felt that they had 'done a good job' or cared for someone as they would wish to be cared for. These sentiments are expressed in the following data extract which summarizes most of the elements required for a quality outcome:

We were able to provide a very high standard of care I feel ** and his family thought so too. We enabled $* *$ to stay at home to die where he wanted to be. He was pain free and dignified and we achieved that by working with the hospice. Any voluntary support I could muster round any support, we used everybody. We had a truly multidisciplinary approach to this man's care. And it worked very well. His wife was very supportive and of course the patient was. It was nice; it was a privilege to look after that man because everything was set up really well. It was a nice home to work in. Nice in the sense that his wife was willing to accept people into her home. Because it is difficult isn't it when you know it's your home and you've got all these people and I think it was - I am blowing my own trumpet really - good planning. It was, I felt as if I did a good job in planning and anticipating well what they might need and it paid off.

\section{Patient and carer satisfaction}

It has often been said that the ultimate test of quality relates to whether the patient and carer are satisfied with the care provided. To complement our work with home nurses we sought to gain a service user perspectives on the palliative care services received by people with a terminal illness (Beaver et al., 1999). We interviewed terminally ill patients, their carers and bereaved carers. Cleary this is a very sensitive area in which to work, nevertheless we were able to glean valuable information. It is noteworthy that the quality of communication was an important factor in judging the quality of care received, the value of effective communication goes beyond merely avoiding the wrongs that arise from poor communication. Many participants identified talking, and support that was implicit in this as a major beneficial input from health professionals. The quality of care was often judged on the basis of personal touches rather than technical intervention. Nurses who called in when they were passing, in addition to routine visits, and family doctors who gave their home phone numbers, even though no participant used it, had a resoundingly positive effect. One could conclude that knowing the patient first hand is possible in palliative care situations and has primacy over technological care. This may partially explain why many home care nurses enjoy working in a palliative care situation.

\section{Conclusion}

It is apparent that community nurses, patients and carers were able to articulate clearly the essential components of high quality palliative care and the circumstances which militate against the ideal. Whilst a number of key factors were identified, forming a good rapport with the patient and family was viewed as essential by all. This is not a new finding, but important to reiterate in the context of the provision of palliative care by community nurses. Given the pivotal role played by establishing a strong relationship, the early referral of patients to the community nursing service is of primary importance. Occasions when this did not occur could prove problematic because it would mean that the nurse was intervening in a crisis situation with no prior knowledge of the patient. Community nurses regularly act as care coordinators or mangers orchestrating the involvement of other services. There was a widely held perception that most patients in our study wanted to die at home and if this was not achieved then care was deemed to have fallen short. Relationships with Family Doctors (GPs) were critical 
for a number of reasons. First, in the early referral of patients to the community nursing service; second, in maintaining contact with community nurses to avoid unnecessary hospital admissions and third, for symptom control purposes. Spending time with patients requiring palliative care was highly valued by nurses and patients alike. Whilst palliative care is an area of work which home/community nurses have always valued it has achieved greater significance in the UK following the transfer of some nursing duties to social services, since it permits qualified community nurses the luxury of providing holistic care. It is worthy of note, that despite medical advances and changes in the organization of care, the factors associated with achieving good quality palliative care have changed little over the years. Similarly the work of Glaser and Strauss and Donabedian are as relevant today as helpful frameworks for interpreting nursing work as they were at their inception in the 1960s. The Structure, Process and Outcome approach highlighted in this presentation could be viewed as the first stage in the development of benchmarks for a quality review of palliative care provided by community nurses. Whilst this standard might be viewed as a useful building block, as opposed to a comprehensive list, it is of particular interest given the 'bottom-up' approach. Further studies examining the perspectives of others namely patients, carers and specialist nurses and doctors are needed to see the full picture.

\section{Future research}

In conclusion the research base of community nursing is weak and research findings from hospital settings are usually applied in the home without further testing. There is a need to promote programmatic work in the area of improving the quality of patient care. There are few studies which work towards identifying new and improved interventions. In the last 40 years, in terms of evaluation methods and models we have not in scientific terms moved very far forward, rather we have had a play on words and a variation of themes. It is hoped that the next decade will accelerate the pace of scientific progress in this area, as research and clinical governance arrangements promote large scale programmatic work rather than a proliferation of more small scale studies completed primarily for higher degrees.

\section{Acknowledgements}

The article draws upon a programme of work conducted with a number of collaborators. A debt of gratitude is owed to the following people: Lynn Austin, Kinta Beaver, Ann Caress, Christine Hallett and Shaun Speed. Without their hard work and expertise the research findings included in this article would not have been generated.

\section{References}

Austin, L., Luker, K. Caress, A. and Hallett, C. 2000: Palliative care: community nurses' perceptions of quality. Quality in Health Care 9, 151-58.

Beaver, K., Luker, K. and Woods, S. 1999: The views of terminally ill people and lay carers on primary care services. International Journal of Palliative Nursing 5, 266-74.

Donabedian, A. 1969: Some issues in evaluating the quality of nursing care. American Journal of Public Health 59, 1833-36.

Glaser, B.E. and Strauss, A.L. 1965: Awareness of dying. New York: Aldine.

Luker, K.A., Austin, L., Caress, A. and Hallett, C. 2000: The importance of 'knowing the patient': community nurses' constructions of quality in providing palliative care. Journal of Advanced Nursing 31, 775-82.

Ohrui, T. 2004: Caregivers murder the frail elderly in Japan. Letter BMJ, 4th June.

Sibbald, B., Shen, J. and McBride, A. 2004: Changing the skillmix of the health care workforce. Journal of Health Service Research Policy 9, 28-38.

Speed, S. and Luker, K.A. 2004: Changes in patterns of knowing the patient: the case of British District Nurses. International Journal of Nursing Studies 41, 921-31. 